\title{
Mistargeted protein disrupts mitochondrial metabolism in inherited Fanconi syndrome
}

As proximal tubule cells have high energy requirements, mitochondrial dysfunction has been suggested as a possible cause of impaired proximal tubular function in Fanconi syndrome. Consistent with this hypothesis, Richard Warth, William A. Gahl, Robert Kleta and colleagues recently identified a mutation in a five-generation family with isolated autosomal-dominant Fanconi syndrome that leads to disruption of mitochondrial metabolism and impaired proximal tubular function.

Using genome-wide linkage analysis and gene sequencing,

the researchers identified this heterozygous missense mutation in the EHHADH gene in all affected family members. EHHADH encodes peroxisomal bifunctional enzyme (PBE), which has a role in fatty acid oxidation and is strongly expressed in the proximal tubules.

"Surprisingly, complete inactivation of PBE in mice did not cause any renal phenotype," says Warth. This finding suggests a dominant-negative effect of the mutation on proximal tubular function rather than haploinsufficiency.

The researchers used permanently transfected, inducible proximal tubular cell lines to investigate the disease-causing effects of the mutation, which was predicted to create a new mitochondrial-targeting motif in PBE. They report that mutant PBE localized mainly to mitochondria, whereas nonmutant PBE localized only to peroxisomes. The mistargeted protein associated with enzymes involved in mitochondrial fatty acid oxidation - the primary energy source in proximal tubules-and impaired energy production. "Like a wrong cogwheel in a gearbox, mutant PBE interferes with mitochondrial function," explains Warth.

Proximal tubule cells that expressed mutant PBE versus the nonmutant protein had a reduced capacity for transepithelial substrate transport, a key tubular function.

"In agreement with mitochondrial dysfunction being the disease-causing mechanism, levels of mitochondrial metabolites were increased in urine samples from the family members who had Fanconi syndrome," says Warth. "Our findings indicate that a monogenic defect resulting in intracellular mistargeting of a mutant protein can cause mitochondrial damage and isolated organ disease-in the case of this family, renal Fanconi syndrome."

Ellen F. Carney

Original article Klootwijk, E. D. etal. Mistargeting of peroxisomal EHHADH and inherited renal Fanconi's syndrome. N. Engl. J. Med. 370, 129-138 (2014) 